PROCEEDINGS OF THE

AMERICAN MATHEMATICAL SOCIETY

Volume 127, Number 6, Pages 1753-1760

S 0002-9939(99)05094-7

Article electronically published on February 11, 1999

\title{
ON THE ASYMPTOTIC STABILITY IN FUNCTIONAL DIFFERENTIAL EQUATIONS
}

\author{
A. O. IGNATYEV
}

(Communicated by Hal L. Smith)

\begin{abstract}
Consider a system of functional differential equations $d x / d t=$ $f\left(t, x_{t}\right)$ where $f$ is the vector-valued functional. The classical asymptotic stability result for such a system calls for a positive definite functional $V(t, \varphi)$ and negative definite functional $d V / d t$. In applications one can construct a positive definite functional $V$, whose derivative is not negative definite but is less than or equal to zero. Exactly for such cases J. Hale created the effective asymptotic stability criterion if the functional $f$ in functional differential equations is autonomous ( $f$ does not depend on $t$ ), and N. N. Krasovskii created such criterion for the case where the functional $f$ is periodic in $t$. For the general case of the non-autonomous functional $f \mathrm{~V}$. M. Matrosov proved that this criterion is not right even for ordinary differential equations. The goal of this paper is to prove this criterion for the case when $f$ is almost periodic in $t$. This case is a particular case of the class of non-autonomous functionals.
\end{abstract}

\section{INTRODUCTION}

Let $x=\left(x_{1}, \ldots, x_{n}\right) \in R^{n}, t \in R,|x|=\sqrt{x_{1}^{2}+\cdots+x_{n}^{2}}$. For a given $h>0, C$ denotes the space of continuous functions mapping $[-h ; 0]$ into $R^{n}$ and for $\varphi \in C$, $\|\varphi\|=\sup _{-h \leq \theta \leq 0}|\varphi(\theta)|$. According to [4] we denote

$$
C_{H}=\{\varphi \in C:\|\varphi\| \leq H\} .
$$

If $x$ is a continuous function of $u$ defined on $-h \leq u<A, A>0$, and if $t$ is a fixed number satisfying $0 \leq t<A$, then $x_{t}$ denotes the restriction of $x$ to the segment $[t-h ; t]$ so that $x_{t}$ is an element of $C$ defined by $x_{t}(\theta)=x(t+\theta)$ for $-h \leq \theta \leq 0$.

Consider a system of functional differential equations

$$
\frac{d x}{d t}=f\left(t, x_{t}\right)
$$

and obtain conditions on a Lyapunov functional to insure that the zero solution is asymptotically stable.

In this system $d x / d t$ denotes the right-hand derivative of $x$ at $t, t$ is time, and $f(t, \varphi) \in R^{n}$ is defined on $[0 ; \infty) \times C_{H} ; f(t ; 0) \equiv 0$.

Received by the editors September 12, 1997.

1991 Mathematics Subject Classification. Primary 34K20.

Key words and phrases. Functional differential equations, Lyapunov functionals, asymptotic stability.

(C)1999 American Mathematical Society 
According to [4] we denote by $x\left(t_{0}, \varphi\right)$ a solution of (1) with initial condition $\varphi \in C_{H}$ where $x_{t_{0}}\left(t_{0}, \varphi\right)=\varphi$, and we denote by $x\left(t, t_{0}, \varphi\right)$ the value of $x\left(t_{0}, \varphi\right)$ at $t$ and $x_{t}\left(t_{0}, \varphi\right)=x\left(t+\theta, t_{0}, \varphi\right),-h \leq \theta \leq 0$.

It is assumed that the vector-valued functional $f(t, \varphi)$ is continuous on $[0 ; \infty) \times$ $C_{H}$ so that a solution will exist for each continuous initial condition. For continuation of solutions, we suppose that $f$ takes closed bounded sets of $[0 ; \infty) \times C_{H}$ into closed bounded sets of $R^{n}$.

Let $V(t, \varphi)$ be a continuous functional defined for $t \geq 0, \varphi \in C_{H}$. The upper right-hand derivative of $V$ along solutions of (1) is defined to be [9]

$$
\frac{d V\left(t, x_{t}\left(t_{0}, \varphi\right)\right)}{d t}=\varlimsup_{\Delta t \rightarrow+0}\left\{V\left(t+\Delta t, x_{t+\Delta t}\left(t_{0}, \varphi\right)\right)-V\left(t, x_{t}\left(t_{0}, \varphi\right)\right)\right\} \frac{1}{\Delta t} .
$$

If $V$ satisfies a Lipschitz condition in the second argument, then this limit is uniquely determined.

The classical criterion of asymptotic stability of zero solution of equations (1), which was obtained by N. N. Krasovskii [10], assumes the existence of a positive definite functional $V$ and a negative definite functional $d V / d t$. In applications one can constract a positive definite functional $V$, whose derivative is not negative definite but is less than or equal to zero. Exactly for such cases J. Hale [8] created the effective asymptotic stability criterion if the functional $f$ in equations (1) is autonomous ( $f$ does not depend on $t$ ), and N. N. Krasovskii [10] created such criterion for the case where the operator $f$ is periodic in $t$. For the general case of the non-autonomous operator $f \mathrm{~V}$. M. Matrosov [12] proved that this criterion is not right even for ordinary differential equations. The goal of this paper is to prove this criterion for the case when $f$ is almost periodic in $t$. This case is a particular case of the class of non-autonomous operators.

\section{Definitions And PRELIMINARY RESUlts}

Definition 1 ([1], [2], [3], [5], [6], [11], [14], [15]). A continuous function $F(t)$ : $R \rightarrow R^{n}$ is called almost periodic if for every $\varepsilon>0$ there exists $l=l(\varepsilon)>0$ such that any segment $[\alpha ; \alpha+l], \alpha \in R$, contains at least one number $\tau$ such that $|F(t+\tau)-F(t)|<\varepsilon$ for every $t \in R$. A number $\tau$ is called an $\varepsilon$-almost period of $F$.

Let us introduce the following definition which is analogous to [11], [14].

Definition 2. A continuous functional $F(t, \varphi): R \times C_{r} \rightarrow R^{n}(0<r<\infty)$ is called uniformly almost periodic in $t$ if for every $\varepsilon>0$ there exists $l=l(\varepsilon, r)>0$ such that any segment $[\alpha ; \alpha+l], \alpha \in R$, contains at least one number $\tau$ such that $|F(t+\tau, \varphi)-F(t, \varphi)|<\varepsilon$ for every $t \in R, \varphi \in C_{r}$.

Remark. A continuous function $F(t)$, which satisfies Definition 1 is called uniformly almost periodic in papers [1], [2], [3], [11], so Definitions 1 and 2 are somewhat different from corresponding Definitions in [1], [2], [3], [11].

Lemma 1 ([11]). Let $F_{1}(t), \ldots, F_{N}(t): R \rightarrow R^{n}$ be almost periodic functions. Then for every $\varepsilon>0$ there exists $l=l(\varepsilon)>0$ such that any segment $[\alpha ; \alpha+l], \alpha \in R$, contains a number $\tau$ such that

$$
\left|F_{i}(t+\tau)-F_{i}(t)\right|<\varepsilon, \quad i=1,2, \ldots, N ; t \in R .
$$


We denote

$$
\begin{array}{r}
C_{H(L)}=\left\{\varphi \in C_{H}:\left|\varphi\left(x_{1}\right)-\varphi\left(x_{2}\right)\right| \leq L\left|x_{1}-x_{2}\right|\right. \\
\text { for each } \left.x_{1}, x_{2} \in[-h ; 0]\right\} \subset C_{H} .
\end{array}
$$

Lemma 2. If the functional $F(t, \varphi): R \times C_{H(L)} \rightarrow R^{n}$ is Lipschitzian in $\varphi$ and almost periodic in $t$ for every fixed $\varphi \in C_{H(L)}$, then it is uniformly almost periodic in $t$.

Proof. Since the functional $F(t, \varphi)$ satisfies Lipschitz conditions in $\varphi$, then

$$
|F(t, \varphi)-F(t, \psi)| \leq L_{1}\|\varphi-\psi\|
$$

where $L_{1}$ is the Lipschitz constant.

Let $\varepsilon>0$ be any real number. $C_{H(L)}$ is the set of uniformly bounded equicontinuous functions, therefore $C_{H(L)}$ is a compact set. Hence there is a finite set of functions $\varphi_{1}, \ldots, \varphi_{N}$ such that $\varphi_{j} \in C_{H(L)}(j=1, \ldots, N)$ and for each $\varphi \in C_{H(L)}$ there exists a number $i(1 \leq i \leq N)$ such that

$$
\left\|\varphi-\varphi_{i}\right\|<\frac{\varepsilon}{3 L_{1}} \text {. }
$$

From Lemma 1 it follows that there exists $l>0$ such that in any segment $[\alpha ; \alpha+l]$ there exists a number $\tau$, such that

$$
\left|F\left(t, \varphi_{i}\right)-F\left(t+\tau, \varphi_{i}\right)\right|<\frac{\varepsilon}{3}
$$

for each $t \in R, i=1, \ldots, N$.

We will now show that for every $\varphi \in C_{H(L)}$, each number $\tau$, which satisfies inequality (4), is an $\varepsilon$-almost period of the functional $F(t, \varphi)$. Let $\varphi_{k}$ be the same element of the set $\varphi_{1}, \ldots, \varphi_{N}$ for which $\left\|\varphi-\varphi_{k}\right\|<\varepsilon /\left(3 L_{1}\right)$. Then from (2)-(4) we obtain

$$
\begin{gathered}
|F(t+\tau, \varphi)-F(t, \varphi)| \leq\left|F(t+\tau, \varphi)-F\left(t+\tau, \varphi_{k}\right)\right| \\
+\left|F\left(t+\tau, \varphi_{k}\right)-F\left(t, \varphi_{k}\right)\right|+\left|F\left(t, \varphi_{k}\right)-F(t, \varphi)\right| \\
<\frac{\varepsilon}{3}+2 L_{1} \cdot \frac{\varepsilon}{3 L_{1}}=\varepsilon .
\end{gathered}
$$

The inequality (5) proves Lemma 2.

\section{MAin Results}

In this section we consider the system of functional differential equations (1) under the assumptions above. We also assume that the functional $f(t, \varphi)$ is Lipschitzian in $\varphi$ and almost periodic in $t$ for every fixed $\varphi \in C_{H}$.

Lemma 3. Consider the solution $x\left(t_{0}, \varphi_{0}\right)$ of the system (1). We suppose that $x_{t}\left(t_{0}, \varphi_{0}\right)$ belongs to $C_{r}(0<r<H)$ for $t \geq 0$. Let $\left\{\varepsilon_{k}\right\}$ be a monotonically approaching zero sequence of positive numbers and $\left\{\tau_{k}\right\}$ a sequence of $\varepsilon_{k}$-almost periods of $f(t, \varphi)$ (for every $\varepsilon_{k}$ there corresponds an $\varepsilon_{k}$-almost period $\tau_{k}$ ). Then the limit relation

$$
\lim _{k \rightarrow \infty}\left\|x_{t^{*}}\left(t_{0}, \varphi_{k}\right)-x_{t^{*}+\tau_{k}}\left(t_{0}, \varphi_{0}\right)\right\|=0
$$

holds, where $\varphi_{k}=x_{t_{0}+\tau_{k}}\left(t_{0}, \varphi_{0}\right)$ and $t^{*}$ is a fixed moment of time which is more than $t_{0}\left(t^{*}>t_{0}\right)$. 
Proof. Consider the solutions of the system (1)

$$
x\left(t_{0}, \varphi_{k}\right)
$$

and

$$
x\left(t_{0}+\tau_{k}, \varphi_{k}\right) .
$$

For the time $\Delta t=t^{*}-t_{0}$ the function $\varphi_{k}$ moves to the function $x_{t^{*}}\left(t_{0}, \varphi_{k}\right)$ along the trajectory (7) and $\varphi_{k}$ moves to the function

$$
x_{t^{*}+\tau_{k}}\left(t_{0}+\tau_{k}, \varphi_{k}\right)=x_{t^{*}+\tau_{k}}\left(t_{0}, \varphi_{0}\right)
$$

along the solution (8). The restriction of the solution $x$ of the system (1), $x_{t}\left(t_{0}+\tau_{k}, \varphi_{k}\right)$, with initial boundary value problem $\varphi_{k}=x_{t_{0}+\tau_{k}}$ may be interpreted as one of the system

$$
\frac{d x}{d t}=f\left(t+\tau_{k}, x_{t}\right)
$$

with initial function $\varphi_{k}$ and initial moment of time $t_{0}$. If $t$ is large enough, then $x_{t} \in C_{H(L)}$. But according to Lemma 2 the right-hand side of the system (1) is uniformly almost periodic in $t$ on the set $R \times C_{H(L)}$, therefore the right-hand sides of the systems (1), (9) differ from each other no matter how small, if $k$ is a large enough natural number. Hence the limit relation (6) follows.

Theorem 1. Let functional differential equations (1) satisfy the above conditions. There exists a continuous functional $V(t, \varphi): R \times C_{H} \rightarrow R$ which is locally Lipschitz in $\varphi$ such that the following conditions are fulfilled on the set $R \times C_{H}$ :

(i) $a(|\varphi(0)|) \leq V(t, \varphi) \leq b(\|\varphi\|)$, where $a, b \in K ; K$ is a class of Hahn's functions [7], [13];

(ii) $V(t, \varphi)$ is almost periodic in $t$ for each fixed $\varphi \in C_{H}$;

(iii) $d V / d t \leq 0, d V / d t \not \equiv 0$ on each solution of the system (1).

Then the solution

$$
x=0
$$

of functional differential equations (1) is asymptotically stable.

Proof. From conditions (i), (iii) it follows that the solution (10) is uniformly stable [8], [9]. Let $\varepsilon \in(0 ; H)$ be any positive number. Denote by $t_{0} \in R$ the initial moment of time. By the stability of the zero solution there exists $\delta>0$ such that if $\varphi \in C_{\delta}$, then $x_{t}\left(t_{0}, \varphi\right) \in C_{\varepsilon}$ for every $t \geq t_{0}$. Choose such a $\delta>0$ and show that any solution $x\left(t_{0}, \varphi\right)$ with $\varphi \in C_{\delta}$ tends to zero as $t \rightarrow \infty$. Suppose that this is not true, i.e. there exist $\eta>0$ and $\varphi_{0} \in C_{\delta}$ such that $\left|x\left(t, t_{0}, \varphi_{0}\right)\right|>\eta>0$ as $t \geq t_{0}$.

The function $V(t)=V\left(t, x_{t}\left(t_{0}, \varphi_{0}\right)\right)$ is monotonically non-increasing because $d V / d t \leq 0$. Hence there exists the limit

$$
\lim _{t \rightarrow \infty} V(t)=\lim _{t \rightarrow \infty} V\left(t, x_{t}\left(t_{0}, \varphi_{0}\right)\right)=V_{0} \geq a(\eta)>0
$$

and it is easy to see that $V\left(t, x_{t}\left(t_{0}, \varphi_{0}\right)\right) \geq V_{0}$ for $t \in\left[t_{0} ; \infty\right)$.

Consider some monotonically approaching zero sequence $\left\{\varepsilon_{k}\right\}$ of positive numbers, where $\varepsilon_{1}$ is sufficiently small. By Lemma 2 for every $\varepsilon_{i}$ there exists a sequence 
of $\varepsilon_{i}$-almost periods $\tau_{i, 1}, \tau_{i, 2}, \ldots, \tau_{i, n}, \ldots \rightarrow \infty$ for functionals $f(t, \varphi)$ and $V(t, \varphi)$, such that inequalities

$$
\begin{gathered}
\left|V\left(t+\tau_{i, n}, \varphi\right)-V(t, \varphi)\right|<\varepsilon_{i}, \\
\left|f\left(t+\tau_{i, n}, \varphi\right)-f(t, \varphi)\right|<\varepsilon_{i}
\end{gathered}
$$

hold for each $t \in R, \varphi \in C_{\varepsilon}$. Without loss of generality one can suppose $\tau_{i, n}<\tau_{i+1, n}$ for every $i, n$. Designate $\tau_{k}=\tau_{k, k}$.

Consider the sequence of functions $\varphi_{k}=x_{t_{0}+\tau_{k}}\left(t_{0}, \varphi_{0}\right) \quad(k=1,2, \ldots)$. It is a bounded sequence of equicontinuous functions because $\varphi_{k} \in C_{\varepsilon}$, therefore there is a limit function $\varphi^{*}$ of this sequence. Without loss of generality one can assume the sequence $\varphi_{k}$ itself converges to $\varphi^{*}$. Because of continuity and almost periodicity of the functional $V(t, \varphi)$ we obtain

$$
\begin{gathered}
V\left(t_{0}, \varphi^{*}\right)=\lim _{n \rightarrow \infty} V\left(t_{0}, \varphi_{n}\right) \\
=\lim _{k \rightarrow \infty} \lim _{n \rightarrow \infty} V\left(t_{0}+\tau_{k}, \varphi_{n}\right) \\
=\lim _{n \rightarrow \infty} V\left(t_{0}+\tau_{n}, \varphi_{n}\right) \\
=\lim _{n \rightarrow \infty} V\left(t_{0}+\tau_{n}, x_{t_{0}+\tau_{n}}\left(t_{0}, \varphi_{0}\right)\right)=V_{0} .
\end{gathered}
$$

Now consider the solution $x\left(t_{0}, \varphi^{*}\right)$. From the condition (iii) of the theorem, the existence of such a moment of time $t^{*}\left(t^{*}>t_{0}\right)$ follows, when the inequality

$$
V\left(t^{*}, x_{t^{*}}\left(t_{0}, \varphi^{*}\right)\right)=V_{1}<V_{0}
$$

holds.

Solutions of functional differential equations (1) are continuous in initial data, so one can write

$$
\lim _{k \rightarrow \infty}\left\|x_{t^{*}}\left(t_{0}, \varphi_{k}\right)-x_{t^{*}}\left(t_{0}, \varphi^{*}\right)\right\|=0
$$

because

$$
\lim _{k \rightarrow \infty}\left\|\varphi_{k}-\varphi^{*}\right\|=0
$$

Hence it follows that

$$
\lim _{k \rightarrow \infty} V\left(t^{*}, x_{t^{*}}\left(t_{0}, \varphi_{k}\right)\right)=V_{1}
$$

Using the uniform almost periodicity property of $f(t, \varphi)$ and the limit relation (6), we obtain the inequality

$$
\left\|x_{t^{*}}\left(t_{0}, \varphi_{k}\right)-x_{t^{*}+\tau_{k}}\left(t_{0}, \varphi_{0}\right)\right\| \leq \gamma_{k}
$$

where $\gamma_{k} \rightarrow 0$ as $k \rightarrow \infty$. Because of the uniform almost periodicity property of $V(t, \varphi)$ we have

$$
\left|V\left(t^{*}, \varphi\right)-V\left(t^{*}+\tau_{k}, \varphi\right)\right|<\varepsilon_{k}
$$

for every $\varphi \in C_{H}$, and from conditions (11), (12) it follows that

$$
\left|V\left(t^{*}, x_{t^{*}+\tau_{k}}\left(t_{0}, \varphi_{0}\right)\right)-V_{1}\right|<\eta_{k},
$$

where $\eta_{k} \rightarrow 0$ as $k \rightarrow \infty$. 
From (13) we obtain

$$
\left|V\left(t^{*}, x_{t^{*}+\tau_{k}}\left(t_{0}, \varphi_{0}\right)\right)-V\left(t^{*}+\tau_{k}, x_{t^{*}+\tau_{k}}\left(t_{0}, \varphi_{0}\right)\right)\right|<\varepsilon_{k} .
$$

From (14), (15) we have

$$
\left|V\left(t^{*}+\tau_{k}, x_{t^{*}+\tau_{k}}\left(t_{0}, \varphi_{0}\right)\right)-V_{1}\right|<\eta_{k}+\varepsilon_{k},
$$

where $\eta_{k}+\varepsilon_{k} \rightarrow 0$ as $k \rightarrow \infty$.

On the other hand

$$
\lim _{k \rightarrow \infty} V\left(t^{*}+\tau_{k}, x_{t^{*}+\tau_{k}}\left(t_{0}, \varphi_{0}\right)\right)=V_{0}
$$

The relations (16), (17) are in contradiction to the inequality $V_{1}<V_{0}$. This contradiction proves Theorem 1.

Theorem 2. Let the right-hand side of functional differential equations (1) be such that there exists a continuous functional $V(t, \varphi): R \times C_{H} \rightarrow R$ which is locally Lipschitz in $\varphi$ such that the following conditions are fulfilled on the set $R \times C_{H}$ :

(i) $|V(t, \varphi)| \leq b(\|\varphi\|), b \in K$;

(ii) $V(t, \varphi)$ is almost periodic in $t$ for each fixed $\varphi \in C_{H}$;

(iii) for every $t \in R$ and for every $\delta>0$ there exists $\varphi \in C_{\delta}$, such that $V(t, \varphi)>0$;

(iv) $d V / d t \geq 0 ; d V / d t \not \equiv 0$ on each solution of the system (1).

Then the solution (10) of functional differential equations (1) is unstable.

Proof. Let $\varepsilon \in(0 ; H)$. We shall take arbitrary $t_{0} \in R$ and arbitrary small $\delta>0$. Let us choose $\varphi_{0} \in C_{\delta}$ such that $V\left(t_{0}, \varphi_{0}\right)>0$. We can do it by condition (iii) of the theorem. By the condition (i) there exists $\eta>0$ such that $|V(t, \varphi)|<$ $V\left(t_{0}, \varphi_{0}\right)$ for every $\varphi \in C_{\eta}$. The function $V(t)=V\left(t, x_{t}\left(t_{0}, \varphi_{0}\right)\right)$ is nondecreasing, i.e. $V\left(t, x_{t}\left(t_{0}, \varphi_{0}\right)\right) \geq V\left(t_{0}, \varphi_{0}\right)$ for $t \geq t_{0}$. It means that $\left\|x_{t}\left(t_{0}, \varphi_{0}\right)\right\| \geq \eta$ for each $t \geq t_{0}$. We shall show that there exists a moment of time $t_{1}\left(t_{1}>t_{0}\right)$, such that $\left\|x_{t_{1}}\left(t_{0}, \varphi_{0}\right)\right\|>\varepsilon$. Suppose that this is not true, i.e. inequalities

$$
\eta \leq\left\|x_{t}\left(t_{0}, \varphi_{0}\right)\right\| \leq \varepsilon
$$

hold for each $t>t_{0}$.

Using inequalities (18) and the condition (iv) of the theorem, we obtain a contradiction by means of the same way as in the proof of Theorem 1. We omit the literal repetition of these reasonings. The contradiction proves that the semitrajectory $x\left(t_{0}, \varphi_{0}\right)$ leaves $C_{\varepsilon}$. The proof is complete.

Example. Consider the non-linear equation

$$
\begin{aligned}
\frac{d x}{d t}= & -2 x^{3}(t)+4 x^{2}(t) x(t-h) \\
& +\left(-12+3 \sin ^{2}(\sqrt{2} t)+3 \sin ^{2} t\right) x(t) x^{2}(t-h)+4 x^{3}(t-h)
\end{aligned}
$$


and the functional

$$
V\left(t, x_{t}\right)=\frac{1}{2} x^{2}(t)+\int_{t-h}^{t} x^{4}(\theta) d \theta
$$

Its time derivative $d V / d t$ along the solutions of (19) is

$$
\begin{aligned}
\frac{d V}{d t}= & \frac{1}{2} \cdot 2 x(t)\left[-2 x^{3}(t)+4 x^{2}(t) x(t-h)\right. \\
& \left.\quad+\left(-12+3 \sin ^{2}(\sqrt{2} t)+3 \sin ^{2} t\right) x(t) x^{2}(t-h)+4 x^{3}(t-h)\right] \\
+ & x^{4}(t)-x^{4}(t-h) \\
= & -2 x^{4}(t)+4 x^{3}(t) x(t-h)+\left(-12+3 \sin ^{2}(\sqrt{2} t)+3 \sin ^{2} t\right) x^{2}(t) x^{2}(t-h) \\
+ & 4 x(t) x^{3}(t-h)+x^{4}(t)-x^{4}(t-h) \\
= & -x^{4}(t)+4 x^{3}(t) x(t-h)-6 x^{2}(t) x^{2}(t-h)+4 x(t) x^{3}(t-h)-x^{4}(t-h) \\
+ & \left(-6+3 \sin ^{2}(\sqrt{2} t)+3 \sin ^{2} t\right) x^{2}(t) x^{2}(t-h) \\
& =-[x(t)-x(t-h)]^{4}-3\left(2-\sin ^{2}(\sqrt{2} t)-\sin ^{2} t\right) x^{2}(t) x^{2}(t-h) .
\end{aligned}
$$

For any $\varepsilon>0$ small enough there exists a sequence $t_{1}, t_{2}, \ldots, t_{n}, \cdots \rightarrow+\infty$ such that

$$
0<2-\sin ^{2}\left(\sqrt{2} t_{i}\right)-\sin ^{2} t_{i}<\varepsilon \quad(i=1,2, \ldots) .
$$

The right-hand side of the equation (19) is not periodic in $t$. Hence we cannot apply Krasovskii's corresponding theorems on the asymptotic stability, but we can use Theorem 1 because the right-hand side of (19) is almost periodic in $t$ and $d V / d t<0$ for each $t>0$. Therefore the zero solution of (19) is asymptotically stable.

\section{REFERENCES}

1. M. S. Berger and Y. Y. Chen, Forced Quasiperiodic and Almost Periodic Solution for Nonlinear Systems, Nonlinear Analysis 21 (12) (1993), 949-965. MR 95b:34066

2. A. S. Besicovitch, Almost Periodic Functions, Dover, New York, 1954. MR 16:817a

3. H. Bohr, Almost Periodic Functions, Chelsea, New York, 1947. MR 8:512a

4. T. A. Burton, Uniform Asymptotic Stability in Functional Differential Equations, Proceedings of the American Mathematical Society 68 (2) (1978), 195-199. MR 58:149

5. C. Corduneanu, Almost Periodic Functions, 2nd edition, Chelsea Publ. Co., New York, 1989.

6. A. M. Fink, Almost Periodic Differential Equations. Lecture Notes in Math., vol. 377, Springer-Verlag, Berlin - Heidelberg - New York, 1974. MR 57:792

7. W. Hahn, Stability of Motion, Springer, New York - Berlin - Heidelberg, 1967. MR 36:6716

8. J. Hale, Theory of Functional Differential Equations, Springer - Verlag, New York - Heidelberg - Berlin, 1977. MR 58:22904

9. V. B. Kolmanovskii and V. R. Nosov, Stability of Functional Differential Equations, Academic Press, New York, 1986. MR 88e:34001

10. N. N. Krasovskii, Stability of Motion, Stanford University Press, Stanford, California, 1963. MR 26:5258

11. B. M. Levitan and V. V. Zhikov, Almost Periodic Functions and Differential Equations, Cambridge University Press, Cambridge, 1982. MR 84g:34004

12. V. M. Matrosov, On the Theory of Stability, Prikladnaya Matematika i Mekhanika 26 (4) (1962), 992-1002 (Russian); English transl., J. Appl. Math. Mech. 26 (1962), 1506-1522. MR 27:403

13. N. Rouche, P. Habets, and M. Laloy, Stability Theory by Liapunov's Direct Method, SpringerVerlag, New York, 1977. MR 56:9008 
14. G. Seifert, On uniformly almost periodic sets of functions for almost periodis differential equations, Tôhoku Math.J., The Second Series 34 (2) (June 1982), 301-309. MR 84k:34053

15. T. Yoshizawa, Stability Theory and the Existence of Periodic Solutions and Almost Periodic Solutions, Appl. Math. Sciences, vol. 14, Springer - Verlag, Berlin - Heidelberg - New York, 1975. MR 57:6673

Institute for Applied Mathematics \& Mechanics, R. Luxemburg Street, 74, Donetsk340114, UKRAINE

E-mail address: ignat@iamm.ac.donetsk.ua 